\title{
A Performance Analysis of Movement Patterns
}

\author{
Corina Sas ${ }^{1}$, Gregory O'Hare ${ }^{1}$, and Ronan Reilly ${ }^{2}$ \\ 1 University College Dublin, Belfield, Dublin 4, Ireland \\ 2 National University of Ireland, Maynooth, Co. Kildare, Ireland
}

\begin{abstract}
This study investigates the differences in movement patterns followed by users navigating within a virtual environment. The analysis has been carried out between two groups of users, identified on the basis of their performance on a search task. Results indicate significant differences between efficient and inefficient navigators' trajectories. They are related to rotational, translational and localised-landmarks behaviour. These findings are discussed in the light of theoretical outcomes provided by environmental psychology.
\end{abstract}

\section{Introduction}

An understanding of how people explore an indoor virtual space can provide not only theoretical contributions but can also be harnessed within practical applications. Designing flexible Virtual Environments (VEs) which are able to adapt themselves in order to support user navigation is one of the most promising application fields. Designing adaptive VEs for navigation support necessitates sensitivity to differing types of users. Such adaptive VEs should be able to discriminate between different groups of users, who require different accommodations. These groups of users differ not only in their performance on spatial tasks, but also in their spatial behaviour. Another significant aspect in the design of adaptive VEs for navigation support is the identification of ways to accommodate individual differences in navigational patterns.

This paper focuses on the analysis of users' spatial behaviour, as reflected in their trajectory paths. Movement paths allow an online and unobtrusive identification of user groups. Trajectory classification offers potential in performing such kinds of identification [6]. The analysis of movement paths also allows the implicit extraction of navigational patterns embedded in trajectory paths.

\section{Study Design}

The experiments have been carried out within a desktop VE [3], which due to its tractable characteristics permitted us to record the users' positions and headings at each moment in time. Adopting a physical world metaphor the VE consists of a virtual multi-story building where each one of the levels contains three rooms. Its projection has a rectangular shape of $16 \times 27$ virtual metres. The ECHOES environment comprises a virtual multi-story building, each one of 


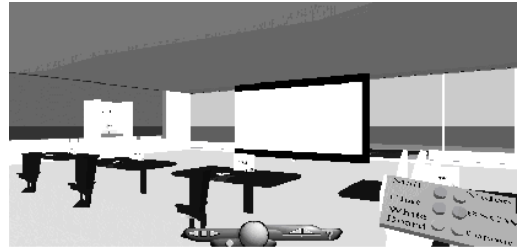

Fig. 1. Virtual Training Room

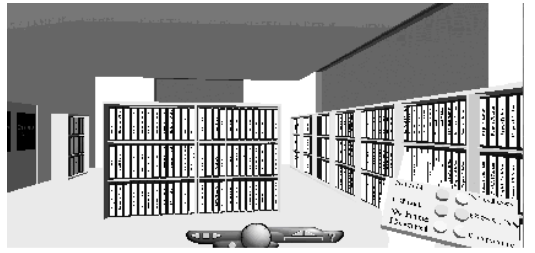

Fig. 2. Virtual Library

the levels containing several rooms: conference room, training room (Figure 1), library (Figure 2), lobby etc.

There is no predefined set of paths, such as halls or corridors which would limit the user choice of movements. The users can move freely in the space, freedom limited only by the walls and objects located within the spatial layout. Users can navigate in terms of moving forwards, backwards or rotating, through the use of the directional keys. They merely use the mouse for selecting a new floor on the panel located in the virtual lift.

The study involved three phases: familiarisation, exploration and performance measurement. Initially, users were allowed to become accustomed with the VE and to learn movement control. After this, they were asked to perform an exploration task. The exploration task within the virtual building lasts for approximately 25 minutes. After the completion of this task, during which participants acquired (implicitly) spatial knowledge related to the VE, they were tested. Users were placed on the third level and asked to find a particular room located on the ground floor of the virtual building (the library). The time needed to accomplish this task acted as an indicator of the level of spatial knowledge acquired within the VE: the shorter the search time, the better the spatial knowledge [5]. According to the time required for the search task, users have been identified as low spatial users, when they needed significantly longer time to find the library (Mean $=49$ seconds), or high spatial users who found the library straight away (Mean $=7$ seconds). Within this paper, the terms of low versus high spatial users are related to this particular outcome and they also capture the dichotomy between poor versus good or inefficient versus efficient navigators.

The sample consisted of 32 students from the Department of Computer Science in University College Dublin and volunteers were paid for their participation.

The following sections focus on aspects supporting differentiation between efficient and inefficient navigators' trajectories. They are related to rotational, translational and localised-landmarks user behaviour during navigation.

\subsection{Rotational Behaviour}

Given the significance of rotations along a movement path, we analysed these in order to identify differences which discriminate efficient from inefficient spatial 


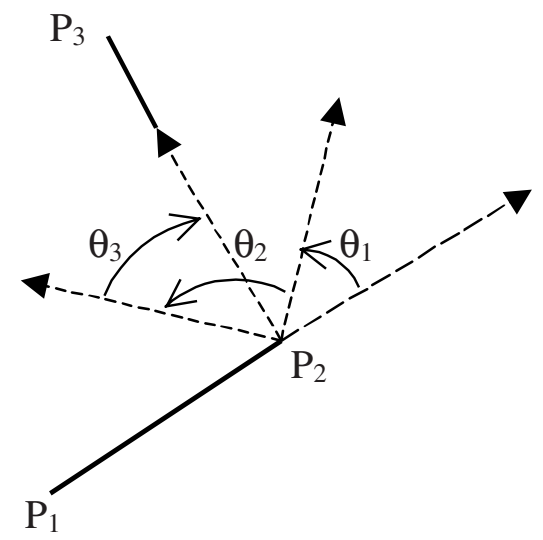

Fig. 3. Observation Angle

behaviour. Each action performed by the user is either a rotation or a translation. Rotations receive increased attention, since they represent those joints, where trajectory segments are articulated at different angles. The location where rotation is performed and the rotation angle provide valuable information about the user's orientation and user's intentions.

Each angle performed at a particular location, possibly consisting of one or more successive rotations, has been computed and averaged for each user. This angle we entitle observation angle since it allows the user to observe the environment through the increased view field it facilitates. Figure 3 presents a sample of user's behaviour, where $\mathrm{P}_{1}, \mathrm{P}_{2}$ and $\mathrm{P}_{3}$ represent three consecutive positions in time, while $\theta_{1}, \theta_{2}$ and $\theta_{3}$ represent there consecutive changes of heading, carried out at location $\mathrm{P}_{2}$.

The observation angle was computed as follows:

$$
\text { Observation angle }=\sum_{i=1}^{k}\left|\theta_{i}\right|,
$$

where $k$ is the number of successive rotations (in our example $k=3$ ).

A $t$-test suggests significant difference between the values of these angles corresponding to rotations performed by efficient versus inefficient users. Thus, low spatial users perform significantly higher changes of heading (Mean above $65^{\circ}$ ) compared to high spatial users (Mean below 45 $)(t(12)=1.92, p<0.05$ ).

When the sign of the rotation angle has been also considered, another angle indicator was obtained. It represents the angle between two adjacent segments of trajectory which suggests a change of direction between two translations. Such an angle is referred to as moving angle and differs from the previous indicator when users change the rotation direction within a set of consecutive rotations. Such a moving angle between consecutive translations performed at different headings is equal or smaller than the observation angle. Figure 4 presents the 


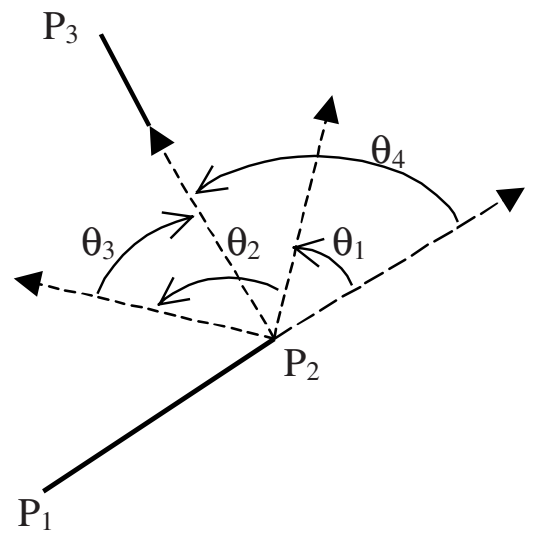

Fig. 4. Moving Angle

same sample of user's behaviour as that depicted in Figure 3 where the moving angle is the angle $\theta_{4}$.

Findings suggest that low spatial users performed significantly higher changes of heading (Mean above $50^{\circ}$ ) when measured as moving angles, compared to high spatial users (Mean below $\left.35^{\circ}\right)(t(12)=1.83, p<0.05)$. Looking at the distribution of these angles, it appeared that low spatial users performed significantly more rotations higher than $90^{\circ}(61.2 \%)$, compared to high spatial users $(38.8 \%)$, (continuity correction: $\chi^{2}(1)=20.71, p<0.05$ ).

The analysis of spatial behaviour with respect to the number of rotations and translations within the entire set of trajectories, revealed another interesting finding. On average per trajectory, high spatial users performed a significantly higher number of rotations (Mean $=12.07$ ) than low spatial users (Mean = $10.36),(t(11178)=10.98, p<0.001)$. When this analysis was restricted to the numbers of successive rotational steps performed between two translations, it appeared that high spatial users performed significantly less such kinds of consecutive rotations (Mean $=2.23$ ), compared to low spatial users (Mean = $3.01),(t(10947)=9.93, p<0.001)$. Such successive rotational steps compose a larger change of heading performed in a given location.

\subsection{Translational Behaviour}

High spatial users performed also significantly less consecutive translations $($ Mean $=1.60)$ than low spatial users (Mean $=1.80),(t(11514)=3.92, p<$ $0.001)$. High spatial users performed also significantly more translations per trajectory $($ Mean $=11.97)$ compared to low spatial users $($ Mean $=10.26),(t(11192)$ $=10.90, p<0.001)$. In addition, the length of each straight line segment, namely the distance covered through consecutive translations, was measured along each trajectory. Such segments have been obtained as a result of consecutive translations performed by the user. A $t$-test indicated significant differences with respect 
to the average length of the straight line segments between high spatial users $($ Mean $=2.94)$ and low spatial users $($ Mean $=3.82)(t(12)=2.49, p<0.05)$.

\subsection{Behaviour Around Landmarks}

An important aspect related to translations refers to behaviour around landmarks. Are there any differences in the way efficient and inefficient navigators visit or revisit landmarks, in the way they navigate along a given landmark, or to what distance from the landmarks of interest they navigate? These are the kind of questions that this section tries to answer. Without being statistically significant, low spatial users visit less landmarks (Mean $=11.25)$ then high spatial users $($ mean $=16.27)(t(12)=1.50, \mathrm{p}>0.05)$.

An important outcome suggests that low spatial users revisited significantly more time the same landmarks (Mean $=6.53$ ), as opposed to high spatial users $($ Mean $=3.60),(t(12)=2.95, p<0.05)$. Without reaching significance, other findings suggest that high spatial users visited more rooms (Mean $=9$ ) than low spatial users (Mean $=6.93$ ). Findings also indicate that high spatial users move along the nearest landmarks, significantly longer (Mean $=20.88$ events), compared to low spatial users (Mean $=14.31$ events) $(t(12)=1.97, p<0.05)$. In doing so it seems that such landmarks act as some form of attractor. $T$-test analysis indicates that low spatial users move along the landmarks of interest at significantly longer distances (Mean $=1.92$ ), compared to high spatial users $($ Mean $=1.68),(t(12)=1.95, p<0.05)$. This implies that the search performed by high spatial users is more systematic, focusing thoroughly on one landmark at the time. Once a particular landmark acts as an attractor and a complete search has been performed in its vicinity, the need for revisiting it decreases significantly.

There is no significant difference between trajectories performed by high versus low spatial users in terms of the trajectory length. Given this result, it would be interesting to investigating whether the same relationship holds with respect to the area covered by high and low spatial users.

\section{Discussion}

Results of this study suggest that the poor trajectories are characterised by more turns at sharp angles, more changes of direction and significantly more straight line segments. The key outcome of this study is that compared to inefficient spatial behaviour, the efficient spatial behaviour can be interpreted as an example of the Minimum Energy Principle. The minimum energy principle expresses itself through those procedural and strategic rules which enable users to achieve the goals with a minimum investment of resources, both temporal and cognitive [7].

The efficiency of spatial behaviour performed by high spatial users is reflected also in the fact that these users try to grasp the entire picture, rather than focusing on its details. Thus, they visit each level of the building, usually in a systematic manner, then they visit more rooms and within the rooms, more 
landmarks than low spatial users. These users are interested in acquiring a larger and more synthetic view of the virtual building, rather than an analytic one. Such a top down approach in exploring the virtual building is complemented by a thorough search in the vicinity of each landmark of interest.

In addition, the way in which high spatial users search around landmarks differs from that employed by low spatial users. The same holistic approach employed at a macro level can be identified: high spatial users perform a thorough search around the landmarks which are considered of interest. They perform significantly more translations and rotations around these landmarks, at shorter distances and accordingly, they revisit these landmarks less.

Spatial orientation refers to users' knowledge of position and orientation within the VE 12]. Through their behaviour, high spatial users succeeded in maintaining a better spatial orientation. Spatial orientation refers to users' knowledge of position and orientation within the VE [12]. High spatial users usually maintain the same main direction or steady orientation, only smoothly altered through small turns. They avoid both long straight movements and large changes of direction, thus maintaining the course of movement.

On the other hand, trajectories performed by low spatial users contain more changes of directions of great angles. These users change their direction not only more often but also more drastically, with serious negative impact on the spatial orientation.

The rotational behaviour of low spatial users presents several major limitations. These users rotate more often at angles greater than $90^{\circ}$, for both observing and changing the movement orientation. They perform significantly more consecutive rotations which dramatically increase the risk of disorientation.

Findings suggest that high spatial users carry out significantly more rotations and translations along a trajectory (on average 12 as opposed to 10), but significantly less such consecutive events (on average 2 rotations as opposed to 3 ). The actions of high spatial users seem to be more frequent, evenly distributed in time, and much smaller (both in changing the heading and changing the location). This is an important outcome which explains how the efficient navigators are more successful in maintaining their orientation and subsequently acquire better spatial knowledge. Within Table 1 we have codified a set of rules which may effectively be used to differentiate between efficient navigators and inefficient navigators within VEs.

\section{Conclusions}

This paper investigates individual differences in navigating in VEs. Findings indicate that there are individual differences in navigational patterns, which are related to performance on spatial tasks, or in other words with users' spatial abilities.

Efficient navigators maintain the movement direction and avoid sharp angles. Low spatial users significantly violate the Minimum Energy Principle which ad- 
Table 1. Low Level Navigational Rules

\begin{tabular}{|c|c|}
\hline Efficient Rules & Inefficient Rules \\
\hline \multicolumn{2}{|c|}{ Rotations } \\
\hline More small rotations & Fewer small rotations \\
\hline Fewer great rotations & More great rotations (sharp angles) \\
\hline Fewer changes of heading $\left(>90^{\circ}\right)$ & More changes of heading $\left(>90^{\circ}\right)$ \\
\hline $\begin{array}{l}\text { More rotations on average } \\
\text { per trajectory }(12)\end{array}$ & $\begin{array}{l}\text { Fewer rotations on average } \\
\text { per trajectory }(10)\end{array}$ \\
\hline $\begin{array}{l}\text { Fewer successive rotations } \\
\text { in average per trajectory }(2)\end{array}$ & $\begin{array}{l}\text { More successive rotations } \\
\text { in average per trajectory }(3)\end{array}$ \\
\hline \multicolumn{2}{|c|}{ Observational angle } \\
\hline Small changes of heading $\left(<45^{\circ}\right)$ & High changes of heading $\left(>65^{\circ}\right)$ \\
\hline \multicolumn{2}{|c|}{ Moving angle } \\
\hline Small changes of heading $\left(<35^{\circ}\right)$ & High changes of heading $\left(>50^{\circ}\right)$ \\
\hline \multicolumn{2}{|c|}{ Translations } \\
\hline $\begin{array}{l}\text { More translations in average } \\
\text { per trajectory }(12)\end{array}$ & $\begin{array}{l}\text { Fewer translations in } \\
\text { average per trajectory (10) }\end{array}$ \\
\hline $\begin{array}{l}\text { Fewer successive translations } \\
\text { in average per trajectory }(1.6)\end{array}$ & $\begin{array}{l}\text { More successive translations } \\
\text { in average per trajectory }(1.8)\end{array}$ \\
\hline $\begin{array}{l}\text { Shorter segments covered by conse } \\
\text { translations ( } 3 \text { virtual metres) }\end{array}$ & $\begin{array}{l}\text { Longer segments covered by consecutive } \\
\text { translations ( } 4 \text { virtual metres) }\end{array}$ \\
\hline \multicolumn{2}{|c|}{ Behaviour around landmarks } \\
\hline More rooms visited (9) & Fewer rooms visited (7) \\
\hline More landmarks visited (16) & Fewer landmarks visited (11) \\
\hline $\begin{array}{l}\text { More movements near landmarks } \\
\text { of interest ( } 20 \text { events) }\end{array}$ & $\begin{array}{l}\text { Fewer movements near landmarks } \\
\text { of interest ( } 14 \text { events) }\end{array}$ \\
\hline $\begin{array}{l}\text { Closer moves to the landmarks of } \\
\text { interest ( } 1.5 \text { virtual metres })\end{array}$ & $\begin{array}{l}\text { Farther moves from the landmarks of } \\
\text { interest ( } 2 \text { virtual metres) }\end{array}$ \\
\hline
\end{tabular}

vocates the reduction of needless expenditure of energy. They are inefficient and their frequent changes of direction increase the risk of disorientation.

Apart from offering a better understanding of spatial behaviour performed within VEs, in terms of individual differences in spatial knowledge acquisition, the study findings have a significant potential for practical applications. They can be used for elaborating design principles for building adaptive VEs, capable of providing real-time navigation support for low spatial users. This can be achieved through enabling low spatial users' access of the efficient navigational rules, governing behaviour of high spatial users. Thus, differentiating between efficient and inefficient spatial behaviours and their underlying strategies could be exploited through applications dedicated to improve spatial skills, such as adaptive VEs. On-going work is encapsulating such rules in the form of an intelligent navigational support agent, based upon a Belief Desire Intention (BDI) model. Such an agent, upon receipt of various observations from a listener agent, updates its set of beliefs (i.e. Belief(User, fewer_small_rotations)). Based upon such beliefs and combinations thereof the agent may adopt commitments to provide 
support for the navigational process. Examples of such may include degradation of the responsiveness of the mouse or movement keys, introduction of directional cues, or indeed the provision of an embodied navigation assistant in the form of an avatar within the VE 4 .

\section{References}

1. Bowman, D., Davis, E., Badre, A., Hodges, L.: Maintaining spatial orientation during travel in an immersive virtual environment. Presence: Teleoperators and Virtual Environments 8(6) (1999) 618-631

2. Bowman, D.A.: Interaction Techniques for Common Tasks in Immersive Virtual Environments. PhD Thesis, Department of Computer Science, Georgia Institute of Technology (1999)

3. O'Hare, G.M.P., Sewell, K., Murphy, A. and Delahunty, T.: An agent based approach to managing collaborative work within both a virtual environment and virtual community. In: Proceedings of the Workshop on Intelligent Agents for Computer Supported Co-Operative Work: Technologies \& Risks (2000)

4. Sas, C., O'Hare, G., Reilly, R.G.: Virtual environment trajectory analysis: A basis for navigational assistance and scene adaptivity. Future Generation Computer Systems. Special Issue on "Interaction and Visualisation Techniques for Problem Solving Environments" (2004) in press.

5. Sas, C., O'Hare, G.M.P.: Presence and individual differences in virtual environment: Usability study. In Sharp, H., Chalk, P., LePeuple, J., Rosbottom, J. (eds) Proceedings of 16th British HCI Conference volume 2, 50-53, London British Computer Society (2002)

6. Sas, C., O'Hare, G.M.P., Reilly, R.G.: On-line trajectory classification. Lecture Notes in Computer Science, Vol. 2659, 1035-1044, Springer-Verlag, Berlin, (2003)

7. Warnett, L., McGonigle, B.: Unsupervised navigation using an economy principle. In Prince, C.G., Demiris, Y., Marom, Y., Kozima, H., Balkenius, C. (eds) in Proceedings of the Second International Workshop on Epigenetic Robotics: Modeling Cognitive Development in Robotic Systems (2002) 